\title{
SCHOTTKY UNIFORMIZATIONS OF SYMMETRIES
}

\author{
G. GROMADZKI \\ Institute of Mathematics, University of Gdansk, Poland \\ e-mail:greggrom@math.univ.gda.pl \\ and R. A. HIDALGO \\ Departamento de Matemática, Universidad Técnica Federico Santa María, Valparaíso, Chile \\ e-mail: ruben.hidalgo@usm.cl
}

(Received 3 April 2012; revised 3 August 2012; accepted 30 August 2012; first published online 25 February 2013)

\begin{abstract}
A real algebraic curve of genus $g$ is a pair $(S,\langle\tau\rangle)$, where $S$ is a closed Riemann surface of genus $g$ and $\tau: S \rightarrow S$ is a symmetry, that is, an anti-conformal involution. A Schottky uniformization of $(S,\langle\tau\rangle)$ is a tuple $(\Omega, \Gamma, P: \Omega \rightarrow S)$, where $\Gamma$ is a Schottky group with region of discontinuity $\Omega$ and $P: \Omega \rightarrow S$ is a regular holomorphic cover map with $\Gamma$ as its deck group, so that there exists an extended Möbius transformation $\widehat{\tau}$ keeping $\Omega$ invariant with $P \circ \widehat{\tau}=\tau \circ P$. The extended Kleinian group $K=\langle\Gamma, \widehat{\tau}\rangle$ is called an extended Schottky groups of rank $g$. The interest on Schottky uniformizations rely on the fact that they provide the lowest uniformizations of closed Riemann surfaces. In this paper we obtain a structural picture of extended Schottky groups in terms of Klein-Maskit's combination theorems and some basic extended Schottky groups. We also provide some insight of the structural picture in terms of the group of automorphisms of $S$ which are reflected by the Schottky uniformization. As a consequence of our structural description of extended Schottky groups, we get alternative proofs to results due to Kalliongis and McCullough (J. Kalliongis and D. McCullough, Orientation-reversing involutions on handlebodies, Trans. Math. Soc. 348(5) (1996), 1739-1755) on orientation-reversing involutions on handlebodies.
\end{abstract}

2010 Mathematics Subject Classification. 30F10, 30F40, 30F50.

1. Introduction. Throughout this Introduction, $S$ will denote a closed Riemann surface of genus $g$. We say that $S$ is symmetric if it admits an anti-conformal automorphism of order two, say $\tau: S \rightarrow S$, called a symmetry of $S$; in this case we say that the pair $(S,\langle\tau\rangle)$ is a real algebraic curve and that the fixed points of the symmetry $\tau$ are the real points of the real algebraic curve. The connected components of fixed points of the symmetry are called the ovals or mirrors of it and, by Harnack's theorem [6], such a number of ovals is at most $g+1$. If the symmetry has fixed points, then it is called a reflection; otherwise it is called an imaginary reflection. The quotient space $R=S /\langle\tau\rangle$ turns out to be a compact (possible bordered) Klein surface, and conversely every compact (possible bordered) Klein surface is obtained in that way. The compact Klein surface $R$ has boundary if and only if $\tau$ is a reflection; in this case the number of boundary components of $R$ is equal to the number of ovals of $\tau$ (see the foundational monograph by Alling and Greenleaf [2]). The theory of real algebraic curves is related to the theory of real forms of complex algebraic curves and its study is nowadays well 
understood. Most of the achievements in this area (see for example [3] viewed as a survey) were possible mainly due to a good understanding of the algebraic structure of non-Euclidean crystallographic (NEC) groups introduced in [15] by Macbeath and the well-developed Teichmüller theory for Fuchsian and NEC groups $[4,16,30]$. We are interested in studying real algebraic curves in terms of uniformizations (see below) of closed Riemann surfaces, but we will restrict ourselves to the lowest ones.

A uniformization of $S$ is a triple $(\Delta, \Gamma, P: \Delta \rightarrow S)$, where $\Delta$ is a planar Riemann surface, $\Gamma$ is a (finitely generated) group of conformal automorphisms of $\Delta$, acting freely and discontinuously on it, and $P: \Delta \rightarrow S$ is a regular covering map with $\Gamma$ as its deck group. By the results given in [19] we may assume, and it will be assumed from now on, that $(\Gamma, \Delta)$ is a function group, that is, $\Gamma$ is a finitely generated Kleinian group and $\Delta$ is a $\Gamma$-invariant connected component of its region of discontinuity. The collection of uniformizations of $S$ is partially ordered: If $\left(\Delta_{1}, \Gamma_{1}, P_{1}: \Delta_{1} \rightarrow S\right)$ and $\left(\Delta_{2}, \Gamma_{2}, P_{2}: \Delta_{2} \rightarrow S\right)$ are two uniformizations of $S$, then the first is higher than the second if there is a continuous map $h: \Delta_{1} \rightarrow \Delta_{2}$ so that $P_{1}=P_{2} \circ h$ (note that $h$ is necessarily a holomorphic cover map). In the case that $h$ is a homeomorphism, these two uniformizations are called topologically equivalent (in fact, they are holomorphically equivalent). The highest ones are produced when $\Delta$ is simply connected; for instance, if $g \geq 2$, then a highest uniformization is given if $\Gamma$ is a Fuchsian group (Fuchsian uniformizations) and the lowest ones are produced when $\Gamma$ is a Schottky group (Schottky uniformizations) [19, 21-24].

Let $S$ be a closed Riemann surface and $H$ be a finite group of (conformal/anticonformal) automorphisms of $S$. Let us consider some uniformization $(\Delta, \Gamma, P$ : $\Delta \rightarrow S$ ) of $S$. We say that $H$ lifts with respect to the given uniformization if for every $h \in H$ there exists an automorphism of $\Delta$, say $k: \Delta \rightarrow \Delta$, so that $h \circ P=P \circ k$. The lifted group, say $K$, contains $\Gamma$ as a normal subgroup so that $K / \Gamma$ is isomorphic to $H$ (in particular, if $\Gamma$ is not the trivial group, then $K$ is an infinite group; so it is not isomorphic to $H$ ). In this definition we are not requiring the liftings to be either Möbius or extended Möbius transformations. In the case each of the liftings can be chosen to be either Möbius or extended Möbius transformations, so the lifted group $K$ is a (possible extended) function group, we say that the above is a uniformization of $(S, H)$. A necessary and sufficient condition for $H$ to lift is given in [12] (see Theorem 3.2 in Section 3 for the case of Schottky uniformizations). If the uniformization is a highest uniformization (that is, $\Delta$ is simply connected), then $H$ lifts. By the uniformization theorem, we may assume that $\Delta$ is either the Riemann sphere $\widehat{\mathbb{C}}$, the complex plane $\mathbb{C}$ or the unit disc $\mathbb{D}$ if $g$ is equal to 0,1 or at least 2 respectively. Under such assumption on $\Delta$, the above is a uniformization of $(S, H)$ and the structure of the corresponding extended function group $K$ is well understood. On the other extreme if the uniformization is a lowest one (that is, $\Gamma$ is a Schottky group of rank $g$ and $\Delta$ is its region of discontinuity), then it is not in general true that $H$ lifts with respect to the given Schottky uniformization. Anyway, if $H=\langle\tau\rangle$, where $\tau$ is a symmetry of $S$, it is well known that it is always possible to find a suitable Schottky uniformization for which $\langle\tau\rangle$ lifts [7, 28, 29] (this is a consequence of the fact that there exists a finite collection of pairwise simple loops on $S$, which is invariant under $\tau$ and that cut off $S$ into planar surfaces). Let us assume that $\langle\tau\rangle$ lifts with respect to the above Schottky uniformization. As the region of discontinuity of a Schottky group is of class $O_{A D}$ (that is, it admits no holomorphic function with finite Dirichlet norm [1, p. 241]), every conformal (respectively, anti-conformal) automorphism of the region of discontinuity of a Schottky group is a Möbius (respectively, extended Möbius) 
transformation. In particular, one again obtains a uniformization for $(S,\langle\tau\rangle)$, and the corresponding extended function group $K$ has $\Gamma$ as its orientation-preserving half; we say that $K$ is an extended Schottky group of rank $g$.

A general structural decomposition theorem for function groups, in the sense of Klein-Maskit's combination theorems [20, 24, 25], was stated by Maskit [17, 21-23] in terms of some basic function groups. In the case of extended function groups one has a similar general structural result; in [10] the details and the subtle modifications to Maskit's arguments for the structural description of extended function groups can be found. As the structural picture of Schottky groups is quite simple, we expect to have a more explicit structural picture of an extended Kleinian group $K$ containing a Schottky group as a finite index subgroup; this is mainly due to the absence of (accidental) parabolic elements, that Schottky groups have no elliptic transformations and the fact that the limit set is totally disconnected. In this paper we restrict ourselves to the case when $K$ is an extended Schottky group (see Theorem 2.1) and relate such a structural picture to group of automorphisms of the uniformized surface by its orientation-preserving half Schottky group. Our structural picture of extended Schottky groups can be used to obtain results in [13, Section 7]) about the topological classification of orientation-reversing involutions on handlebodies, whose methods are from combinatorial group theory (finite extensions of free groups) in three-dimensional handlebodies. It seems that their topological methods are not so easy to extend to the classification of more general finite groups of automorphisms of handlebodies, but our methods seem to be easy to generalize, with suitable modifications, to provide the structural picture of (possible extended) function groups $K$ containing a Schottky group $\Gamma$ as a normal subgroup and with quotient $K / \Gamma$ being a finite group. This will be pursued elsewhere, at first for the cyclic case.

In Section 2 we state all our main results. In Section 3 we provide the main definitions (which have not been already stated), the (simple) version of Klein-Maskit's combination theorems we need in this paper and we recall the lifting property of [8] needed in this paper. In Section 4 we provide the proof of the structural picture of extended Schottky groups.

\section{Main results.}

2.1. Basic extended Schottky groups. The basic extended Schottky groups are (i) cyclic groups generated by either a reflection or an imaginary reflection (anti-conformal involution acting freely on the Riemann sphere) or a glide-reflection (extended Möbius transformation whose square is a hyperbolic one) and (ii) real Schottky groups of rank $\mathrm{g}$ : extended Kleinian groups generated by a reflection (called the associated reflection) and a Schottky group of rank $g$ whose elements commute with the associated reflection.

Next we proceed to give a discussion on real Schottky groups. Let $K$ be a real Schottky group, $\Omega$ be its region of discontinuity, $\tau$ be its associated reflection and $\Gamma$ be its associated Schottky group. Clearly, $\Gamma$ is the orientation-preserving half of $K$. If $C$ is the circle of fixed points of $\tau$, then $C$ is invariant under $K$ and the limit set of $K$ is a subset of $C$. Let $D$ be any of the two discs bounded by $C$ and set $C^{0}=C \cap \Omega$. If $D$ is invariant under $\Gamma$, then $\Omega / K=\left(D \cup C^{0}\right) / \Gamma$ is a compact bordered Riemann surface of some genus $p$ with $k>0$ boundary components; we say that the real Schottky group has signature $(+; p ; k)$. If $D$ is not invariant under $\Gamma$, then there is an index two subgroup $K_{0}$ of $K$ that preserves $D$ (it necessarily contains glide-reflections). In fact, 

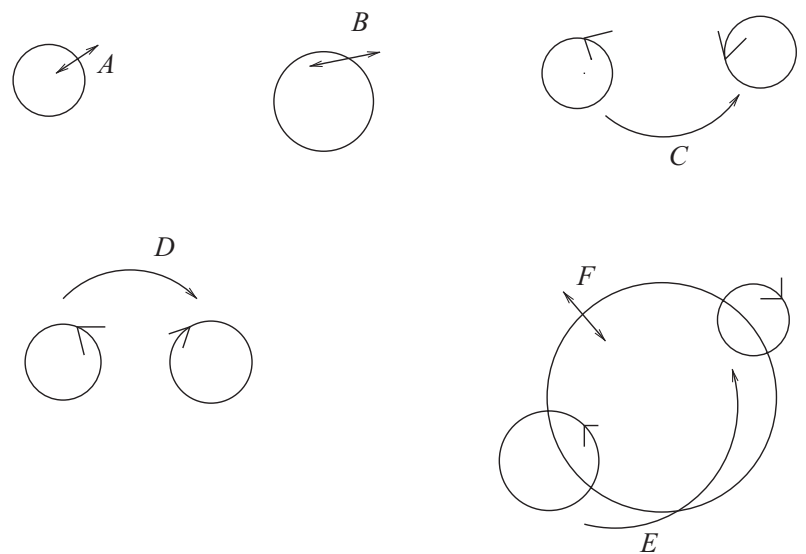

Figure 1. An extended Schottky group $K$ constructed as in Theorem 2.1 with $a=b=c=d=e=r_{1}=1$. In this case $K$ is generated by a reflection $A$, an imaginary reflection $B$, a glide-reflection $C$, a loxodromic $D$ and the real Schottky group generated by the reflection $F$ and the loxodromic transformation $E$. The compact Klein surface $\Omega / K$ is a connected sum of four real projective planes,

a genus one orientable surface and has exactly two boundary components.

let $A_{1}, \ldots, A_{g}$ be a set of free generators of $\Gamma$ and for each $j=1, \ldots, g$, we set $B_{j}$ equal to $A_{j}$ if it keeps invariant $D$ or $B_{j}=\tau \circ A_{j}$ (a glide-reflection) otherwise. Then $B_{1}, \ldots, B_{g}$ generates $K_{0}$ and the quotient $\Omega / K=\left(D \cup C^{0}\right) / K_{0}$ is a compact bordered non-orientable surface, say of topological genus $q$, and $r>0$ boundary components; we say that the real Schottky group has signature $(-; q ; r)$.

2.2. The structural picture of extended Schottky groups. The structural picture of extended Schottky groups in terms of Klein-Maskit's combination theorem (see part (1) in Theorem 3.1 in Section 3) is provided by the following.

THEOREM 2.1 (Structural decomposition of extended Schottky groups). An extended Schottky group can be constructed from Klein-Maskit's combination theorem as the free product of the following basic extended Schottky groups:

(i) Cyclic groups generated by reflections.

(ii) Cyclic groups generated by imaginary reflections.

(iii) Cyclic groups generated by glide-reflections.

(iv) Cyclic groups generated by loxodromic transformations.

(v) Real Schottky groups.

Conversely any group of Möbius and extended Möbius transformations constructed using ' $a$ ' groups of type (i), ' $b$ ' groups of type (ii), 'c' groups of type (iii), ' $d$ ' groups of type (iv) and ' $e$ ' groups of type (v), is an extended Schottky group if and only if $a+b+c+e>0$. If, in addition, the real Schottky groups have ranks $r_{1}, \ldots, r_{e} \geq 1$, then the extended Schottky group has rank $g=a+b+2(c+d)+e-1+r_{1}+\ldots+r_{e}$.

Figure 1 shows an example of the structure of an extended Schottky group of rank 7 constructed as stated in Theorem 2.1. 
REMARK 2.2 Two interesting examples. The following two constructions will be important in the proof of Theorem 2.1. The first example is provided by an HNNextension of a real Schottky group by a cyclic group generated by either a loxodromic transformation or a glide-reflection. The second one is a free product of two real Schottky groups and an HNN-extension of it by a cyclic group generated by either a loxodromic transformation or a glide-reflection.

(1) Let us consider a collection of simple loops on the Riemann sphere, say $\Sigma_{1}, \ldots$, $\Sigma_{2 r+3}$, so that $\Sigma_{3}, \ldots, \Sigma_{2 r+3}$ are circles, $\Sigma_{4}, \ldots, \Sigma_{2 r+3}$ are pairwise disjoint and each one orthogonal to $\Sigma_{3}, \Sigma_{1}$ and $\Sigma_{2}$ are disjoint from all other loops and are separated by the circle $\Sigma_{3}$, and the loops $\Sigma_{1}, \Sigma_{2}, \Sigma_{4}, \ldots, \Sigma_{2 r+3}$ bound a domain $\mathcal{D}$ of connectivity $2 r+2$ (see Figure 2 for the case $r=1$ ). Let $\tau$ be the reflection on $\Sigma_{3}$. For each $j=1, \ldots, r$, let $B_{j}$ be a loxodromic transformation sending the circle $\Sigma_{2 j+2}$ onto the circle $\Sigma_{2 j+3}$, keeping invariant $\Sigma_{3}$ so that $B_{j}(\mathcal{D}) \cap \mathcal{D}=\emptyset$. Let $A$ be either loxodromic or glide-reflection so that $A\left(\Sigma_{2}\right)=\Sigma_{1}$ and $A(\mathcal{D}) \cap \mathcal{D}=\emptyset$ (in particular, its fixed points are separated by $\Sigma_{3}$ ). The group $K_{1}=\left\langle\tau, B_{1}, \ldots, B_{r}\right\rangle$ is a real Schottky group of rank $r$ ( $\tau$ is its associated reflection and $\left\langle B_{1}, \ldots, B_{r}\right\rangle$ is its associated Schottky group of rank $r$ ). The group $K=\left\langle\tau, B_{1}, \ldots, B_{r}, A\right\rangle$ is an extended Kleinian group obtained as the HNNextension of $K_{1}$ by the cyclic group generated by $A$. Note that $K$ is also generated by $K_{1}$ and $T=\tau \circ A$. Let us assume that either $\tau\left(\Sigma_{1}\right)=\Sigma_{2}$ or $\tau\left(\Sigma_{1}\right) \cap \Sigma_{2}=\emptyset$, and that in the case $\tau\left(\Sigma_{1}\right)$ and $\Sigma_{2}$ are disjoint, none of them separates the other from the circle of fixed points of $\tau$. Under such assumptions $T$ is either a reflection or an imaginary reflection (if $\tau\left(\Sigma_{1}\right)=\Sigma_{2}$ ) or a glide-reflection or a loxodromic transformation in the other situation. We may then see that $K$ is an extended Schottky group constructed, as in Theorem 2.1, as a free product of the above real Schottky group $K_{1}$ and a cyclic group of either (i), (ii), (iii) or (iv) type.

(2) Let $\Sigma_{0}$ be a simple loop on the Riemann sphere and let $\Delta_{1}$ and $\Delta_{2}$ be the two open topological discs bounded by it. On $\Delta_{j}$ we consider a collection of circles, say $\Sigma_{3}^{j}, \ldots, \Sigma_{2 r_{j}+3}^{j}$ so that $\Sigma_{4}^{j}, \ldots, \Sigma_{2 r+3}^{j}$ are orthogonal to $\Sigma_{3}^{j}$, all the loops, with the exception of $\Sigma_{3}^{j}$, are pairwise disjoint, and the loops $\Sigma_{4}^{j}, \ldots, \Sigma_{2 r_{j}+3}^{j}$ bound a domain $\mathcal{D}_{j}$ of connectivity $2 r_{j}$. Let $\Sigma_{j}$ be a simple loop contained inside the open disc $\mathcal{E}_{j}$ (the disc bounded by $\Sigma_{3}^{j}$ and disjoint from $\Delta_{3-j}$ ) disjoint from the loops $\Sigma_{4}^{j}, \ldots, \Sigma_{2 r_{j}+3}^{j}$ (see Figure 3 for the case $r_{1}=1$ and $r_{2}=2$ ). Let $\tau_{j}$ be the reflection on $\Sigma_{3}^{j}$. For each $k=$ $1, \ldots, r_{j}$, let $B_{k}^{j}$ be a loxodromic transformation sending the circle $\Sigma_{2 k+2}^{j}$ onto the circle $\Sigma_{2 k+3}^{j}$, keeping invariant $\Sigma_{3}^{j}$ so that $B_{k}\left(\mathcal{D}_{j}\right) \cap \mathcal{D}_{j}=\emptyset$. We also consider a glide-reflection or a loxodromic transformation $E$ so that $E\left(\Sigma_{1}\right)=\Sigma_{2}$ and $E(\mathcal{D}) \cap \mathcal{D}=\emptyset$, where $\mathcal{D}=$ $\mathcal{D}_{1} \cap \mathcal{D}_{2} \cap \mathcal{E}_{1}^{*} \cap \mathcal{E}_{2}^{*}$, and $\mathcal{E}_{j}^{*}=\widehat{\mathbb{C}}-\left(\mathcal{E}_{j} \cup \Sigma_{3}^{j}\right)$. The group $K_{j}=\left\langle\tau_{j}, B_{1}^{j}, \ldots, B_{r_{j}}^{j}\right\rangle$ is a real Schottky group of rank $r_{j}$ (its associated reflection id $\tau_{j}$ and its associated Schottky group of rank $r_{j}$ is $\left.\left\langle B_{1}^{j}, \ldots, B_{r_{j}}^{j}\right\rangle\right)$. Let $K$ be the extended Kleinian group constructed as a free product of $K_{1}$ and $K_{2}$ and an HNN-extension by the cyclic group generated by $E$. It is not difficult to check that $K$ is also generated by $K_{1}$ and $K_{2}$ and the transformation $T=\tau_{2} \circ E \circ \tau_{1}$. Let us assume that the loops $\tau_{1}\left(\Sigma_{1}\right)$ and $\tau_{2}\left(\Sigma_{2}\right)$ are contained in $\mathcal{D}$, that they are either disjoint or the same loop and if they are disjoint, then none of them separates the other from the circle $\Sigma_{3}^{1}$. Under these assumptions, one has that $T$ is either a reflection or an imaginary reflection (in the case $\tau_{1}\left(\Sigma_{1}\right)=\tau_{2}\left(\Sigma_{2}\right)$ ) or a glide-reflection or a loxodromic transformation. So we may see that $K$ is an extended 


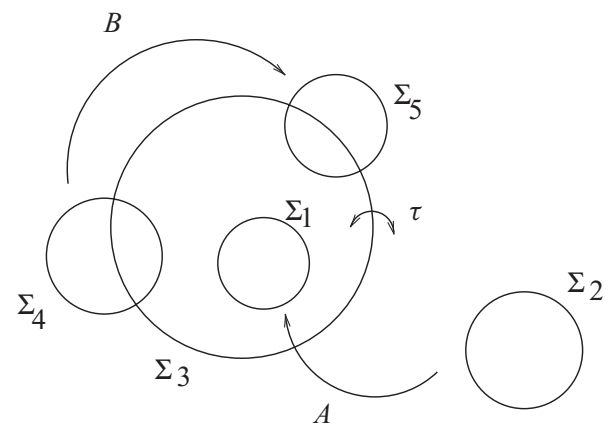

Figure 2. An extended Kleinian group as in (1) of Remark 2.2 with $r=1$.

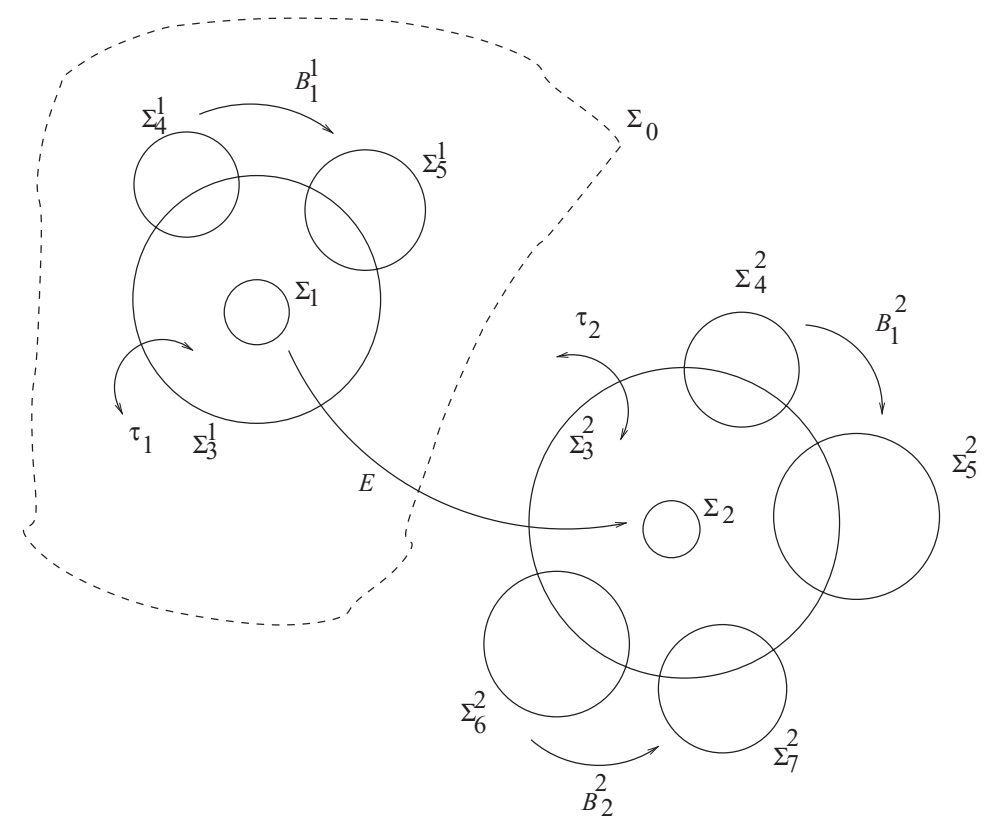

Figure 3. An extended Kleinian group as in (2) of Remark 2.2 with $r_{1}=1$ and $r_{2}=2$.

Schottky group constructed, as in Theorem 2.1, as the free product of two real Schottky groups and a cyclic group of either (i), (ii), (iii) or (iv) type.

2.3. Extended Schottky groups as subgroups of extended Kleinian groups. Let $\widehat{K}$ be an extended Kleinian group containing an extended Schottky group $K$ as a finite index normal subgroup. Let $\Gamma$ be the index two orientation-preserving half of $K$. If $\gamma \in \widehat{K}$ and $A \in \Gamma$, then $\gamma A \gamma^{-1} \in K$ (as $K$ is normal subgroup) and $\gamma A \gamma^{-1}$ preserves the orientation. It follows that $\Gamma$ is also a finite index normal subgroup of $\widehat{K}$. Our second result states that the numbers $a, b$ and $e$ used in the construction of $K$ in Theorem 2.1 can be explicitly computed in terms of the conjugacy classes of the anti-conformal involutions of $\widehat{K}$. Before stating the result we need some definitions. 
REMARK 2.3. We should notice that $\widehat{K}$ may not be in general an extended Schotky group, but if its orientation-preserving half $\widehat{K}^{+}$is torsion free, then $\widehat{K}$ is necessarily an extended Schottky group. In fact, under this assumption, $\widehat{K}^{+}$is a purely loxodromic function group whose region of discontinuity is connected. Now by the classification of function groups in [21, 23], one has $\widehat{K}^{+}$as a Schottky group.

Let $G=\widehat{K} / \Gamma$ (a finite group by our assumptions) and let $\theta: \widehat{K} \rightarrow G$ be the canonical projection with $\operatorname{ker}(\theta)=\Gamma$. If $\tau \in G$ is an involution so that $K_{\tau}=\theta^{-1}(\langle\tau\rangle)$ contains extended Möbius transformations, then $K_{\tau}$ is an extended Schottky group whose orientation-preserving half is $\Gamma$.

Let $\mathcal{C}=\left\{\eta_{i}: i \in I\right\}$ be a maximal collection of anti-conformal involutions (i.e. reflections and imaginary reflections) of $\widehat{K}$ which are non-conjugate in $\widehat{K}$. We call such a set $\mathcal{C}$ a complete set of symmetries of $\widehat{K}$ and we shall refer to its elements as to canonical symmetries.

REMARK 2.4. As the group $\widehat{K}$ is necessarily geometrically finite (since it is a finite extension of a Schottky group), the set $\mathcal{C}$ is finite. However, due to Theorem 2.1, we will not need this fact in the proof of the next theorem and so we do not go into details.

Next, for each $i \in I$ we set $I(i) \subset I$ defined by those $j \in I$ so that $\theta\left(\eta_{i}\right)$ and $\theta\left(\eta_{j}\right)$ are conjugate in $G$ (in particular, $i \in I(i)$ ). Note at this point that it may happen that for $j \in I(i), \eta_{j}$ can be an imaginary reflection even if $\eta_{i}$ is a reflection and vice versa. We set by $J(i)$ the subset of $I(i)$ defined by those $j$ for which $\eta_{j}$ is an imaginary reflection. We also set by $F(i) \subset I(i)-J(i)$ for those $j$ for which $\eta_{j}$ has a finite centralizer in $\widehat{K}$ and $E(i)=I(i)-(J(i) \cup F(i))$. Note that as $K$ has a finite index in $\widehat{K}$, a reflection $\eta \in \widehat{K}$ has an infinite centralizer $\mathrm{C}(\widehat{K}, \eta)$ in $\widehat{K}$ if and only if it has an infinite centralizer in $K$.

THEOREM 2.5. Let $\widehat{K}$ be an extended Kleinian group containing a Schottky group $\Gamma$ as a finite index normal subgroup. Let $G=\widehat{K} / \Gamma$, let $\theta: \widehat{K} \rightarrow G$ be the canonical projection and let $\mathcal{C}=\left\{\eta_{i}: i \in I\right\}$ be a complete set of symmetries of $\widehat{K}$. Then $K_{i}=$ $\theta^{-1}\left(\left\langle\theta\left(\eta_{i}\right)\right\rangle\right)=\left\langle\Gamma, \eta_{i}\right\rangle$ is an extended Schottky group, constructed using $a_{i}$ reflections, $b_{i}$ imaginary reflections and $e_{i}$ real Schottky groups as in Theorem 2.1, where

$$
\begin{aligned}
a_{i} & =\sum_{j \in F(i)}\left[\mathrm{C}\left(G, \theta\left(\eta_{j}\right)\right): \theta\left(\mathrm{C}\left(\widehat{K}, \eta_{j}\right)\right)\right], \\
b_{i} & =\sum_{j \in J(i)}\left[\mathrm{C}\left(G, \theta\left(\eta_{j}\right)\right): \theta\left(\mathrm{C}\left(\widehat{K}, \eta_{j}\right)\right)\right], \\
e_{i} & =\sum_{j \in E(i)}\left[\mathrm{C}\left(G, \theta\left(\eta_{j}\right)\right): \theta\left(\mathrm{C}\left(\widehat{K}, \eta_{j}\right)\right)\right] .
\end{aligned}
$$

REMARK 2.5. It is well known that if $\widehat{K}$ is an extended Kleinian group containing a Schottky group $\Gamma$ of rank $g \geq 2$ as a finite index normal subgroup, then the index of $\Gamma$ in $\widehat{K}$ is at most 24( $g-1)$ [32]. In [9] the structural picture of Kleinian groups containing a Schottky group of maximal index $12(g-1)$ as a normal subgroup (in terms of Klein-Maskit's combination theorems) and the corresponding quotients are provided (see also [33]). From that information, one obtains a concrete picture for $\widehat{K}$ in the maximal index situation and, in particular, information about the symmetries 
of $\widehat{K}$, their centralizers and the quotient groups $\widehat{K} / \Gamma$. So in these cases the use of Theorem 2.5 to compute the values of $a_{i}, b_{i}$ and $e_{i}$ is not so difficult to carry out.

2.4. Counting topological equivalences of extended Schottky groups. The structural picture of extended Schottky groups provided in Theorem 2.1 easily permits to count the number of topologically different extended Schottky groups of a fixed rank. The main point for this counting argument is a certain uniqueness of the decomposition structure. More precisely, assume that one decomposition of the extended Schottky group $K$ uses ' $a$ ' cyclic groups generated by reflections, ' $b$ ' cyclic groups generated by imaginary reflections, ' $c$ ' cyclic groups generated by glide-reflections, ' $d$ ' cyclic groups generated by loxodromic transformations and ' $e$ ' real Schottky groups. Let us consider the hyperbolic 3-orbifold $\mathcal{O}=\mathbb{H}^{3} / K$. The number ' $a$ ' is the number of boundary components of $\mathcal{O}$ homeomorphic to discs; the number ' $b$ ' is the number of points of $\mathcal{O}$ over which it fails to be a manifold; ' $e$ ' is equal to the number of boundary components different from discs (the topological structure of each of these boundary components determines the corresponding real Schottky group); and $d+c$ is determined from the relation $g=a+b+2(c+d)+e-1+r_{1}+\cdots+r_{e}$.

If $b+c>0$, then we may replace each of the loxodromic generators of the ' $d$ ' cyclic groups by glide-reflections, that is, we may assume $d=0$ (this procedure has been used in [11]).

COROLLARY 2.6. The number of topologically different extended Schottky groups of rank $g$ is bounded above by

$$
\begin{aligned}
& {\left[\frac{g+4}{2}\right]\left[\frac{g+5}{2}\right]-2+\sum_{\left(e, r_{1}, \ldots, r_{e}\right) \in \Delta_{g}}} \\
& \quad \times\left(\left[\frac{g+4-e-\sum_{j=1}^{e} r_{j}}{2}\right]\left[\frac{g+5-e-\sum_{j=1}^{e} r_{j}}{2}\right]-\delta_{\left(e ; r_{1}, \ldots, r_{e}\right)}\right) \times \prod_{j=1}^{e}\left[\frac{2+3 r_{j}}{2}\right],
\end{aligned}
$$

where

$$
\Delta_{g}=\left\{\left(e ; r_{1}, \ldots, r_{e}\right): 1 \leq e, 1 \leq r_{1} \leq \cdots \leq r_{e}, e+\sum_{j=1}^{e} r_{j} \leq g+1\right\}
$$

and $\delta_{\left(e ; r_{1}, \ldots, r_{e}\right)}$ is equal to 1 or 2 for $g-e-\sum_{j=1}^{e} r_{j}$ even or odd, respectively. The symbol $[x]$ denotes the maximum integer less or equal to $x$.

Proof. The structural picture of extended Schottky groups given in Theorem 2.1 asserts that the topological class of an extended Schottky group of rank $g$ is completely determined by the number ' $a$ ' of groups of type (i), the number ' $b$ ' of groups of type (ii), the number ' $c$ ' of groups of type (iii), the number ' $d$ ' of groups of type (iv), the number ' $e$ ' of real Schottky groups (and their ranks $r_{j}$ ) and the topological type of each of these (satisfying the conditions given in Theorem 2.1). Without taking care of the topological type of each of the real Schottky groups (just their ranks), we see that such a number is equal to the number of different tuples of the form

$$
\left(a, b, c, d,\left(e ; r_{1}, \ldots, r_{e}\right)\right)
$$


satisfying the following conditions (see the observations previous to the statement of Corollary 2.6):

(1) $a+b+c+e>0$;

(2) $1 \leq r_{1} \leq r_{2} \leq \cdots \leq r_{e}$;

(3) if $b+c>0$, then $d=0$ and

(4) $g=a+b+2(c+d)+e-1+\sum_{j=1}^{e} r_{j}$. on it.

The above number is equal to the desired formula but without the final product

Secondly, for each such tuple $\left(a, b, c, d,\left(e ; r_{1}, \ldots, r_{e}\right)\right)$ we need to count how many topologically non-equivalent groups can be constructed with such a data. For this we just need to note that we only need to compute, for each $j=1, \ldots, e$, the number $N_{j}$ of topologically different real Schottky groups of a fixed rank $r_{j} \geq 1$; this number $N_{j}$ being equal to the number of topologically different symmetries with fixed points that a closed Riemann surface of genus $r_{j}$ may have, that is, $N_{j}=\left[\left(2+3 r_{j}\right) / 2\right]$ (see [14]; this part takes care of the product at the end of the formula). All the above permit to obtain the desired formula. The details are left to the interested reader.

3. Definitions and notations. In this section we review some of the definitions (which have not been stated before), set some notations and recall some technical results that we will need in this paper. Generalities on Kleinian and extended Kleinian groups can be found, for instance, in $[\mathbf{2 4}, \mathbf{2 6}]$.

An extended Möbius transformation is the composition of a Möbius transformation with complex conjugation. It is well known that the conformal automorphisms (respectively, anti-conformal automorphisms) of the Riemann sphere $\widehat{\mathbb{C}}$ are the Möbius transformations (respectively, extended Möbius transformations). We denote by $\widehat{M}$ the group of Möbius and extended Möbius transformations and by $\mathbb{M}$ its index two subgroups of Möbius transformations. The group $\widehat{\mathbb{M}}$ can also be viewed by the Poincaré extension theorem as the group of hyperbolic isometries of the hyperbolic space $\mathbb{H}^{3}$; in this case $\mathbb{M}$ is the group of orientation-preserving ones. Möbius transformations are classified into parabolic, loxodromic (including hyperbolic) and elliptic. Similarly, extended Möbius transformations are classified into pseudo-parabolic (the square is parabolic), glide-reflections (the square is hyperbolic), pseudo-elliptic (the square is elliptic), reflections (of order two admitting a circle of fixed points on $\widehat{\mathbb{C}}$ ) and imaginary reflections (of order two and having no fixed points on $\widehat{\mathbb{C}}$ ). Each imaginary reflection in $\widehat{\mathbb{M}}$ has exactly one fixed point in $\mathbb{H}^{3}$ and this point determines it uniquely. If $K$ is a subgroup of $\widehat{\mathbb{M}}$, not contained in $\mathbb{M}$, then its canonical orientation-preserving subgroup $K \cap \mathbb{M}$, denoted by $K^{+}$, has index two in $K$.

A Kleinian group is a discrete subgroup of $\mathbb{M}$. Similarly, an extended Kleinian group is a discrete subgroup of $\widehat{M}$ necessarily containing extended Möbius transformations. If $K$ is a (extended) Kleinian group, then its region of discontinuity is the (necessarily open) subset $\Omega$ of $\widehat{\mathbb{C}}$ consisting of those points over which $K$ acts discontinuously. If $\Omega \neq \emptyset$, then we say that $K$ is of the second kind; otherwise of the first kind. Note that $K$ is an extended Kleinian group if and only if $K^{+}$is a Kleinian group: both of these with the same region of discontinuity. A function group (respectively, a extended function group) is a pair $(K, \Delta)$, where $K$ is a finitely generated Kleinian group (respectively, a finitely generated extended Kleinian group) and $\Delta$ is a $K$-invariant connected component of its region of discontinuity. 
The decomposition of function groups in the sense of Klein-Maskit's combination theorems is provided in [21-23] and that for extended function group can be seen in [10] (were the subtle modifications of the arguments for function groups are provided). We next state a simple version of Klein-Maskit's combination theorems which is enough for us in this paper.

THEOREM 3.1 (Klein-Maskit's combination theorem [20, 25]).

(1) (Free products) Let $K_{j}$ be a (extended) Kleinian group with region of discontinuity $\Omega_{j}$, for $j=1,2$. Let $\mathcal{F}_{j}$ be a fundamental domain for $K_{j}$ and assume that there is a simple closed loop $\Sigma$, contained in the interior of $\mathcal{F}_{1} \cap \mathcal{F}_{2}$, bounding two discs $D_{1}$ and $D_{2}$, so that for $j=1,2$, the set $\Sigma \cup D_{j} \subset \Omega_{3-j}$ is precisely invariant under the identity in $K_{3-j}$. Then $K=\left\langle K_{1}, K_{2}\right\rangle$ is a (extended) Kleinian group, with fundamental domain $\mathcal{F}_{1} \cap \mathcal{F}_{2}$, which is the free product of $K_{1}$ and $K_{2}$. Every finite-order element in $K$ is conjugated in $K$ to a finite-order element of either $K_{1}$ or $K_{2}$. Moreover, if both $K_{1}$ and $K_{2}$ are geometrically finite, then $K$ is so.

(2) (HNN-extensions) Let $K_{0}$ be a (extended) Kleinian group with region of discontinuity $\Omega$, and let $\mathcal{F}$ be a fundamental domain for $K_{0}$. Assume that there are two pairwise disjoint simple closed loops $\Sigma_{1}$ and $\Sigma_{2}$, both of these contained in the interior of $\mathcal{F}$, so that $\Sigma_{j}$ bounds a disc $D_{j}$ such that $\left(\Sigma_{1} \cup D_{1}\right) \cap\left(\Sigma_{2} \cup D_{2}\right)=\emptyset$ and that $\Sigma_{j} \cup D_{j} \subset \Omega$ is precisely invariant under the identity in $K_{0}$. If $T$ is either a loxodromic transformation or a glide-reflection so that $T\left(\Sigma_{1}\right)=\Sigma_{2}$ and $T\left(D_{1}\right) \cap D_{2}=\emptyset$, then $K=\left\langle K_{0}, f\right\rangle$ is a (extended) Kleinian group, with fundamental domain $\mathcal{F} \cap\left(D_{1} \cup D_{2}\right)^{c}$, which is the $H N N$-extension of $K_{0}$ by the cyclic group $\langle T\rangle$. Every finite-order element of $K$ is conjugated in $K$ to a finite-order element of $K_{0}$. Moreover, if $K_{0}$ is geometrically finite, then $K$ is so.

The Schottky group of rank 0 is just the trivial group. A Schottky group of rank $g \geq 1$ is a Kleinian group $\Gamma$ generated by loxodromic transformations $A_{1}, \ldots, A_{g}$ so that there are $2 g$ disjoint simple loops, $C_{1}, C_{1}^{\prime}, \ldots, C_{g}, C_{g}^{\prime}$ with a $2 g$-connected outside $\mathcal{D} \subset \widehat{\mathbb{C}}$, where $A_{i}\left(C_{i}\right)=C_{i}^{\prime}$ and $A_{i}(\mathcal{D}) \cap \mathcal{D}=\emptyset$, for $i=1, \ldots, g$. The collection of loops $C_{1}, C_{1}^{\prime}, \ldots, C_{g}$ and $C_{g}^{\prime}$ is called a fundamental set of loops for $\Gamma$ with respect to above generators (these groups are the free product of $g$ cyclic loxodromic groups by use of (1) in Klein-Maskit's combination theorem). The region of discontinuity $\Omega$ of a Schottky group $\Gamma$ of rank $g$ is known to be connected and dense in $\widehat{\mathbb{C}}$ and that $S=\Omega / \Gamma$ is a closed Riemann surface of genus $g$. Classical retrosection theorem states that every closed Riemann surface is obtained, up to conformal equivalence, from a Schottky group as above.

It is well known that a Schottky group of rank $g$ can be defined as a purely loxodromic Kleinian group of the second kind which is isomorphic to a free of rank $g$ [18]. It also follows that every hyperbolic structure on the interior of a handlebody whose conformal boundary is a closed Riemann surface (that is a Kleinian structure of the handlebody) is provided by a Schottky group.

The proof of Theorem 2.1 will be based in the following lifting criteria of groups of automorphisms (either conformal or anti-conformal) of Riemann surfaces to Schottky uniformizations.

TheOREM 3.2. [8] Let $(\Omega, \Gamma, P: \Omega \rightarrow S)$ be a Schottky uniformization of the closed Riemann surface $S$ of genus $g \geq 2$ and let $H$ be a group of (conformal/anti-conformal) 
automorphisms of $S$. Then $H$ lifts with respect to the above Schottky uniformization if and only if there is a collection $\mathcal{F}$ of pairwise disjoint simple loops on $S$ such that

(i) each connected component of $S-\mathcal{F}$ is a planar surface;

(ii) $\mathcal{F}$ is invariant under the action of $H$ and

(iii) for each $\alpha \in \mathcal{F}, P^{-1}(\alpha)$ is a collection of pairwise disjoint simple loops in $\Omega$.

REMARK 3.3. We should note that Theorem 3.2 can be seen as a consequence of the Equivariant Loop Theorem [27], whose proof is based on minimal surfaces, that is, surfaces that minimize the area locally. The proof given in [8] only uses arguments proper to (planar) Kleinian groups and the hyperbolic metric.

We make use of Theorem 3.2 for the case that $H$ is a cyclic group generated by a symmetry $\tau$ of $S$, in which case it states a necessary and sufficient condition for the Schottky uniformization of $S$ to be a Schottky uniformization for the real algebraic curve $(S,\langle\tau\rangle)$.

4. Proof of Theorem. 2.1 In what follows a simple loop means a Jordan curve and a circle on the Riemann surface is either an Euclidean circle in the complex plane or the union of an Euclidean line with the point at infinity. Circles have associated a reflection for which the circle is its locus of fixed points.

4.1. First part. We first prove that the extended Kleinian groups constructed as described in Theorem 2.1 are extended Schottky groups indeed. Let us construct an extended Kleinian group as stated in the second part of Theorem 2.1 as follows (see Figure 4). Consider non-negative integers $a, b, c, d, r, s, p_{1}, \ldots, p_{r}$ (where some of them may be zero) and strictly positive integers $k_{1}, \ldots, k_{r}, q_{1}, \ldots, q_{s}, l_{1}, \ldots, l_{s}$, so that $a+b+c+r+s>0$. Set $n=a+b+c+d+r+s$ and $e=r+s$. Let us consider a collection of $n$ pairwise disjoint simple loops, say $\Sigma_{1}, \ldots, \Sigma_{n}$, all of them bounding a connected domain $\mathfrak{D}$ of connectivity $n$. Let $\mathfrak{D}_{j}$ be the open topological disc bounded by the loop $\Sigma_{j}$ which is disjoint from $\mathfrak{D}$.

(i) For each $j=1, \ldots, a$, we consider a circle $T_{j} \subset \mathfrak{D}_{j}$ and let $\tau_{j}$ be the reflection on $T_{j}$.

(ii) For each $j=1, \ldots, b$, we consider a simple loop $N_{j} \subset \mathfrak{D}_{a+j}$ and let $\eta_{j}$ be an imaginary reflection keeping invariant $N_{j}$ and permuting both discs bounded by it.

(iii) For each $j=1, \ldots, c$, we consider two pairwise disjoint simple loops $D_{j}, D_{j}^{\prime} \subset$ $\mathfrak{D}_{a+b+j}$ (so that $D_{j}, D_{j}^{\prime}$ and $\Sigma_{a+b+j}$ bound a common domain of connectivity 3 ). Let $\delta_{j}$ be a glide-reflection so that $\delta_{j}\left(D_{j}\right)=D_{j}^{\prime}$ and $\left.\delta_{j}(\mathfrak{D}) \cap \mathfrak{D}\right)=\emptyset$.

(iv) For each $j=1, \ldots, d$, we consider two pairwise disjoint simple loops $E_{j}, E_{j}^{\prime} \subset$ $\mathfrak{D}_{a+b+c+j}$ (so that $E_{j}, E_{j}^{\prime}$ and $\Sigma_{a+b+c+j}$ bound a common domain of connectivity 3 ). Let $\epsilon_{j}$ be a loxodromic transformation so that $\epsilon_{j}\left(E_{j}\right)=E_{j}^{\prime}$ and $\left.\epsilon_{j}(\mathfrak{D}) \cap \mathfrak{D}\right)=\emptyset$.

(v) For each $j=1, \ldots, r$, we consider real Schottky groups $F_{j}$ of type $\left(+; p_{j} ; k_{j}\right)$, whose associated reflection $\gamma_{j} \in F_{j}$ has its circle of fixed points $L_{j}$ contained in $\mathfrak{D}_{a+b+c+d+j}$, and the closure of the complement of $\mathfrak{D}_{a+b+c+d+j}$ is contained in a fundamental domain for $F_{j}$.

(vi) For each $j=1, \ldots, s$, we consider real Schottky groups $G_{j}$ of type $\left(-; q_{j} ; l_{j}\right)$, whose associated reflection $\theta_{j} \in G_{j}$ has its circle of fixed points $M_{j}$ contained in 

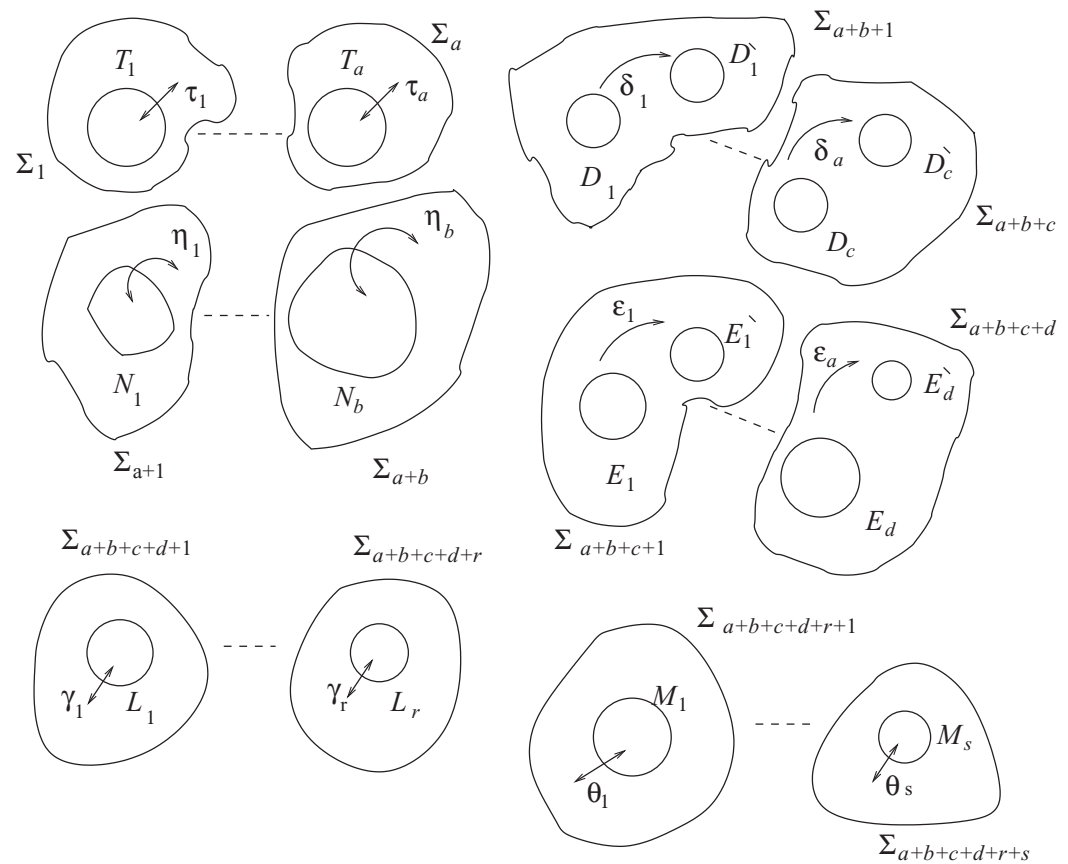

Figure 4. The loops in the geometrical construction.

$\mathfrak{D}_{a+b+c+d+r+j}$, and the closure of the complement of $\mathfrak{D}_{a+b+c+d+r+j}$ is contained in a fundamental domain for $G_{j}$.

The properties of the loops $\Sigma_{j}$ permit to construct (by part (1) of Klein-Maskit's combination theorem 3.1) an extended Kleinian group $K$ as a free product of the $a$ cyclic groups generated by reflections $\tau_{j}$, the $b$ cyclic groups generated by the imaginary reflections $\eta_{j}$, the $c$ cyclic groups generated by the glide-reflections $\delta_{j}$, the $d$ cyclic groups generated by the loxodromic transformations $\epsilon_{j}$ and the $r+s$ real Schottky groups $F_{j}$ and $G_{j}$. We call the type of constructed groups as above a geometrical extended Schottky group of type

$$
\left(\xi ; b, c, d,\left\{p_{1}, \ldots, p_{r}\right\},\left\{q_{1}, \ldots, q_{s}\right\} ; a,\left\{k_{1}, \ldots, k_{r}\right\},\left\{l_{1}, \ldots, l_{s}\right\}\right),
$$

where $\xi=+$ in the case that $b=c=s=0$ (in which case we say that $K$ is orientable) and $\xi=-$ in others cases (in this case we say that $K$ is non-orientable). If $\Omega$ is the region of discontinuity of $K$, then $\Omega / K$ is orientable if and only if $\xi=+$. Lemma 4.1 asserts that $K$ is in fact an extended Schottky group.

Lemma 4.1. A geometrical extended Schottky group $K$ of type

$$
\left(\xi ; b, c, d,\left\{p_{1}, \ldots, p_{r}\right\},\left\{q_{1}, \ldots, q_{s}\right\} ; a,\left\{k_{1}, \ldots, k_{r}\right\},\left\{l_{1}, \ldots, l_{s}\right\}\right)
$$

is an extended Schottky group of rank

$$
\begin{aligned}
g= & a+b+2(c+d)+2 p_{1}+\cdots+2 p_{r}+q_{1}+\cdots+q_{s} \\
& +k_{1}+\cdots+k_{r}+l_{1}+\cdots+l_{s}-1 .
\end{aligned}
$$


Proof. Keeping the above notation, $K$ is generated by $\tau_{1}, \ldots, \tau_{a}, \eta_{1}, \ldots, \eta_{b}$, $\delta_{1}, \ldots, \delta_{c}, \varepsilon_{1}, \ldots, \varepsilon_{d}$ and the real Schottky groups $F_{1}, \ldots, F_{r}$ and $G_{1}, \ldots, G_{s}$. It follows from Klein-Maskit's combination theorems that the finite-order elements in $K$ (different from the identity) are conjugated to one of the generators $\tau_{1}, \ldots, \tau_{a}, \eta_{1}, \ldots$, $\eta_{b}, \gamma_{1}, \ldots, \gamma_{r}$ and $\theta_{1}, \ldots, \theta_{s}$. In this way, we may define a surjective homomorphism

$$
\phi: K \rightarrow\left\langle x: x^{2}=1\right\rangle
$$

by the rule

$$
\begin{gathered}
\phi\left(\tau_{j}\right)=\phi\left(\eta_{j}\right)=\phi\left(\delta_{j}\right)=\phi\left(\gamma_{j}\right)=\phi\left(\theta_{j}\right)=x, \\
\phi\left(\varepsilon_{j}\right)=\phi\left(F_{j}^{+}\right)=\phi\left(K_{j}^{+}\right)=1 .
\end{gathered}
$$

Again, as a consequence of Klein-Maskit's combination theorem, one has that $K$ is geometrically finite and its region of discontinuity $\Omega$ is connected. It follows from the definition of $\phi$ that $K^{+}=\operatorname{ker}(\phi)$ is torsion-free and, in particular, $K^{+}$ is purely loxodromic, geometrically finite function group with connected region of discontinuity $\Omega$. As a consequence of the classification of function groups [23], we obtain that $K^{+}$is a Schottky group and, in particular, $K$ is an extended Schottky group. As $S^{+}=\Omega / K^{+}$is a closed Riemann surface of genus $g$ admitting a symmetry $\tau$ (induced by $K$ ) so that $S^{+} /\langle\tau\rangle=\Omega / K$, we may obtain the value of $g$ as desired.

4.2. Second part. In order to finish the proof of Theorem 2.1, we need to check that every extended Schottky group is in fact a geometrical extended Schottky group.

Let $K$ be an extended Schottky with $\Omega$ as its region of discontinuity. Let $\Gamma=K^{+}$ be its index two Schottky subgroup, say of rank $g, S^{+}=\Omega / \Gamma$ and $\tau: S^{+} \rightarrow S^{+}$be the symmetry induced by $K-\Gamma$ on $S^{+}$. Denote by $P: \Omega \rightarrow S^{+}$the regular covering map whose deck group is the Schottky group $\Gamma$. In this way, $\left(\Omega, \Gamma, P: \Omega \rightarrow S^{+}\right)$is a Schottky uniformization of $\left(S^{+},\langle\tau\rangle\right)$.

As $\Gamma$ has no finite-order elements besides the identity and it has index two in $K$, every finite-order element of $K$, different from the identity, has order two (they are either imaginary reflections of reflections) and every finite-order subgroup of $K$ is either the trivial group or a cyclic group of order two generated by either a reflection or an imaginary reflection. Also, as $\Gamma$ has neither parabolic transformations or elliptic transformations, the circle of fixed points of two different reflections in $\widehat{\Gamma}$ cannot intersect themselves or to be a tangent.

If $C \subset \Omega$ is a simple loop, then we denote by $K_{C}$ its $K$-stabilizer. The condition for $C$ to be contained in the region of discontinuity of $K$ asserts that $K_{C}$ is a finite-order subgroup of $K$ so, as already noted, $K_{C}$ is either trivial or a cyclic group of order two generated by either a reflection or an imaginary reflection.

4.2.1. Construction of a collection of loops on $S^{+}$. Theorem 3.2 asserts the existence of a collection $\mathcal{F}_{0}$ of pairwise disjoint simple loops on $S^{+}$satisfying the following three properties:

(a) The connected components of $S^{+}-\mathcal{F}_{0}$ are planar surfaces.

(b) Each loop in $\mathcal{F}_{0}$ lifts to a loop in $\Omega$ under the covering $P: \Omega \rightarrow S^{+}$. 
(c) The collection $\mathcal{F}_{0}$ is invariant under $\tau$.

LeMma 4.2. We may modify the collection $\mathcal{F}_{0}$, without destroying the properties (a)-(c), in order to assume that it contains all those ovals of $\tau$ which lift to loops under $P: \Omega \rightarrow S^{+}$.

Proof. Let $A \subset S^{+}$be an oval of $\tau$ which lifts to a loop under $P: \Omega \rightarrow S^{+}$and assume that $A$ does not belong to our original collection $\mathcal{F}_{0}$. If no loop in $\mathcal{F}_{0}$ intersects $A$, then clearly we may add $A$ to $\mathcal{F}_{0}$ without destroying properties (a)-(c). If a loop $B \in \mathcal{F}_{0}$ intersects $A$, then we proceed as follows: Let $C, D \subset \Omega$ be simple loops so that $P(C)=A, P(D)=B$ and $C \cap D \neq \emptyset$. We claim that $C \cap D$ consists of two different points. In fact, if $\tau_{C}$ is the reflection on $C$ (so $\tau_{C} \in K$ is a lifting of $\tau$ ), then $\tau_{C}(D)$ belongs to $P^{-1}\left(\mathcal{F}_{0}\right)$ (as such a collection of loops is invariant under $K$ ). But we may see that $D$ and $\tau_{C}(D)$ should intersect over the points in $C \cap D$. It follows that $\tau_{C}(D)=D$. Now the planarity property of $\Omega$ asserts the desired intersection property. Let us denote by $p$ and $q$ the intersection points of $C$ and $D$, by $C^{1}$ one of the two arcs of $C-\{p, q\}$ and by $D^{1}$ and $D^{2}$ the two arcs of $D-\{p, q\}$. Then for $j=1,2$, the loop $D^{j} \cup C^{1} \cup\{p, q\}$ projects to a simple loop $L^{j}$ on $S^{+}$so that $\tau\left(L^{1}\right)=L^{2}$. We may deform continuously the loops $L^{j}$ to simple loops $M^{1}$ and $M^{2}$, respectively, so that they are disjoint from the oval $A$ and also disjoint from all loops in $\mathcal{F}_{0}-\{B\}$, satisfying that $\tau\left(M^{1}\right)=M^{2}$ and that $M^{j}$ lifts to simple loops under $P$. Then we may delete $B$ from $\mathcal{F}_{0}$ and to add the oval $A$ and the loops $M^{1}$ and $M^{2}$. The new collection of loops, in this way constructed, still satisfy properties (a)-(c). Proceeding in this way for each of the ovals of $\tau$ that lifts to loops under $P: \Omega \rightarrow S^{+}$, we produce the desired collection.

As a consequence of Lemma 4.2, we may also assume that our collection $\mathcal{F}_{0}$ satisfies the following extra property.

(d) Each oval of $\tau$ that lifts to a loop under $P: \Omega \rightarrow S^{+}$belongs to $\mathcal{F}_{0}$.

Let $C \subset \Omega$ be a connected component of $P^{-1}\left(\mathcal{F}_{0}\right)$ (a simple loop by (b)). As observed before, (i) $K_{C}$ is trivial or (ii) $K_{C}$ is generated by a reflection $\tau_{C}$ or (iii) $K_{C}$ is generated by an imaginary reflection $\eta_{C}$.

In case (ii) we have the following two possibilities:

(ii.1) the circle $\operatorname{Fix}\left(\tau_{C}\right)$, of fixed points of the reflection $\tau_{C}$, is completely contained in $\Omega$.

(ii.2) $\operatorname{Fix}\left(\tau_{C}\right)$ contains limit points of $\Gamma$.

In case (ii.1) we may see that $P\left(\operatorname{Fix}\left(\tau_{C}\right)\right)$ is an oval of $\tau$. By $(\mathrm{d})$, such an oval belongs to $\mathcal{F}_{0}$. The planarity of $\Omega$ asserts that in this case $C$ and $\operatorname{Fix}\left(\tau_{C}\right)$ should intersect. The disjointness property of the loops in $P^{-1}\left(\mathcal{F}_{0}\right)$ then asserts that $C=\operatorname{Fix}\left(\tau_{C}\right)$.

Let us now assume that we are in case (ii.2). Again, similar to the proof of Lemma 4.2, the intersection between $\operatorname{Fix}\left(\tau_{C}\right)$ and $C$ must consist of two different points, say $p$ and $q$. If one of the two arcs in $\operatorname{Fix}\left(\tau_{C}\right)-\{p, q\}$ is contained in $\Omega$, then we may proceed as in the proof of Lemma 4.2 to replace $P(C)$ with two new loops which are disjoint from any oval of $\tau$ and any other loop in $\mathcal{F}_{0}-\{P(C)\}$, permuted by $\tau$ so that they also lift to loops under $P: \Omega \rightarrow S^{+}$. With this procedure we may assume that each time we have (ii.2), both arcs in $\operatorname{Fix}\left(\tau_{C}\right)-\{p, q\}$ contain limit points, that is, we may assume the following extra property for $\mathcal{F}_{0}$.

(e) The stabilizer in $K$ of loop $C$ in $P^{-1}\left(\mathcal{F}_{0}\right)$ is trivial or generated by an imaginary reflection or a reflection $\tau_{C}$ and, in this last case, either

(e.1) $C=\operatorname{Fix}\left(\tau_{C}\right)$; or 
(e.2) both topological open discs bounded by $C$ contain limit points in $\operatorname{Fix}\left(\tau_{C}\right)$.

Such a constructed collection $\mathcal{F}_{0}$ of loops satisfying (a)-(e) is not necessarily unique. But we may consider one which is minimal in the sense that by deleting any non-empty sub-collection from it some of the properties (a)-(e) do not hold. We still denote such a minimal collection with the symbol $\mathcal{F}_{0}$.

Let $\mathcal{R}$ be a connected components of $\Omega-P^{-1}\left(\mathcal{F}_{0}\right)$. Then $P(\mathcal{R})$ is a planarconnected region of $S^{+}$and $P: \mathcal{R} \rightarrow P(\mathcal{R})$ is a homeomorphism, in particular, $\Gamma$-stabilizer of $\mathcal{R}$ is a finite-order subgroup of $\Gamma$; so trivial. In this way its $K$-stabilizer is either trivial or a cyclic group generated by either a reflection or an imaginary reflection. If the $K$-stabilizer of $\mathcal{R}$ is a cyclic group generated by an imaginary reflection $\eta$, then there should be a simple loop $\delta \subset \mathcal{R}$ so that $\eta(\delta)=\delta$. We may add the loop $P(\delta)$ to $\mathcal{F}_{0}$ without destroying any of the properties (a)-(e). In this way we obtain a new collection of loops, which we denote by the symbol $\mathcal{F}$, satisfying the properties (a)-(e) and also the extra property.

(f) None of the connected components of $\Omega-P^{-1}(\mathcal{F})$ is stabilized by an imaginary reflection of $K$.

REMARK 4.3. As a consequence of the above properties, each connected component of $\Omega-P^{-1}(\mathcal{F})$ has $K$-stabilizer either trivial or a cyclic group generated by a reflection whose circle of fixed points contains limit points of $K$. Moreover, in this last situation, if the reflection is $\rho \in K$ and $C_{\rho}$ is its circle of fixed points, then every loop in $P^{-1}(\mathcal{F})$ intersecting $C_{\rho}$ has the property that each of the two discs bounded by it contains limit points in $C_{\rho}$.

4.2.2. Decomposition of $K$. We call each of the loops in $P^{-1}(\mathcal{F})$ a structure loop of $K$ and each of the connected components of $\Omega-P^{-1}(\mathcal{F})$ a structure region of $K$. As already noted, each structure region is homeomorphic under $P$ to a planar region in $S^{+}$. Moreover, the projection under $P$ of any two structure regions are either equal (both regions are $\Gamma$-equivalent) or disjoint with no common boundary loop or disjoint with some common boundary(ies). Set $S=S^{+} /\langle\tau\rangle$, which a compact Klein surface and let us denote by $Q: S^{+} \rightarrow S$ the two-fold regular branched cover induced by $\tau$. The image under $Q$ of any loop in $\mathcal{F}$ is either a simple loop or a simple arc whose end points belong to the boundary of $S$ (the projection of the ovals of $\tau$ ). As the number of connected components of $S-Q(\mathcal{F})$ is finite, we may choose a finite collection $\mathcal{P}$ of structure regions so that

(1) the projection under $Q$ of any two different structure regions of $\mathcal{P}$ are disjoint; that is, any two structure regions in $\mathcal{P}$ are non-equivalent under $K$;

(2) the union of the projections under $Q$ of all structure regions in $\mathcal{P}$ equals $S-Q(\mathcal{F})$. That is, every structure region is $K$-equivalent to one in $\mathcal{P}$;

(3) the union of the structure regions in $\mathcal{P}$ together with their boundary structure loops is a planar connected domain $\mathcal{D}$ of finite connectivity.

Condition (3) follows from the fact that we may cut off $S$ with some of the loops and $\operatorname{arcs}$ in $Q(\mathcal{F})$ to obtain a planar-connected surface.

If $C$ is a structure loop in the interior of $\mathcal{D}$, then, by (1), its $K$-stabilizer can only be trivial or a cyclic group generated by the reflection whose circle of fixed points contains limit points. In the case the $K$-stabilizer of $C$ is trivial, we may perform part (1) of Klein-Maskit's combination theorem 3.1 for $K$ stabilizers of two structure regions 
having $C$ as a common boundary. Some of the $K$-stabilizers are trivial and others are a cyclic group generated by a reflection whose circle of fixed points contains limit points (at the end, these are to produce the real Schottky groups). In the case that the $K$-stabilizer of $C$ is a cyclic group generated by a reflection, both structure regions having $C$ as a common boundary have $K$-stabilizer the cyclic group generated by the previous reflection. In this case we do not perform any action (just think that the union of these two structure regions together with $C$ is a bigger structure region). So far we have constructed with part (1) of Klein-Maskit's combination theorem 3.1 the free product of a finite collection of cyclic groups generated by some reflections. Denote such a group with the symbol $K_{1}$ (a subgroup of $K$ ).

Next, we should take care of the boundary structure loops of $\mathcal{D}$. Let $C_{1}$ be a boundary structure loop. If the $K$-stabilizer of $C_{1}$ is generated by an imaginary reflection or a reflection with $C_{1}$ as its circle of fixed points, then we may perform part (1) of Klein-Maskit's combination theorem 3.1 between $K_{1}$ and the cyclic group generated by the previous involution (in this case, a simple loop to perform the construction is any loop in a small neighbourhood of $C_{1}$ ). With this type of procedure, we obtain an extended Kleinian group $K_{2}$ (again a subgroup of $K$ ), which is the free product of cyclic groups of types (i) and (ii) in Theorem 2.1 and the group $K_{1}$.

Now we are left those boundary loops with $K$-stabilizer either trivial or a cyclic group generated by a reflection whose circle of fixed points contains limit points of $K$.

Let $C_{2}$ be a boundary loop with $K$-stabilizer to be generated by a reflection $\zeta \in K$ whose circle of fixed points has limit points. Let $R_{1} \in \mathcal{P}$ be the structure region stabilized by $\zeta$ containing $C_{2}$ in the border. The projection loop $Q\left(P\left(C_{2}\right)\right)$ is a simple arc in $S$, whose end points belong to the boundary of $S$. Again, there should be another boundary loop, say $C_{2}^{\prime}$, of $\mathcal{D}$ and either a loxodromic transformation or a glide-reflection $h \in K$ so that $h\left(C_{2}\right)=C_{2}^{\prime}$. The $K$-stabilizer of $C_{2}^{\prime}$ is generated by the reflection $h \circ \zeta \circ h^{-1}$. If $C_{2}^{\prime}$ is also a boundary of $R_{1}$, then $h \circ \zeta \circ h^{-1}=\zeta$ and we perform the HNN-extension of $\langle\zeta\rangle$ by the cyclic group generated by $h$. Next, assume that $h \circ \zeta \circ h^{-1} \neq \zeta$ (that is, $C_{2}^{\prime}$ is not a boundary loop of $R_{1}$ ). Then the structure region $R_{1}$ is neighboured (along $C_{2}$ ) to the structure region $R_{2}$ (which does not belong to $\mathcal{P}$ ) which is $K$-equivalent to the structure region $R_{3}$ in $\mathcal{P}$ stabilized by $h \circ \zeta \circ h^{-1}$ (containing $C_{2}^{\prime}$ in the boundary). We may replace $R_{3}$ with $R_{2}$ without destroying all the previous properties (this is possible since $R_{1}$ and $R_{3}$ are border regions). We may now assume that $h \circ \zeta \circ h^{-1}=\zeta$ and proceed as before with the HNN-extension procedure. In this way, proceeding with all boundary loops of $\mathcal{D}$, which are stabilized by the same $\zeta$, we produce a real Schottky group whose reflection is $\zeta$. We may find a simple loop that separates all the used boundary loops from the rest, we see that the real Schottky group is a free product factor in the HNN-extension of $K_{2}$ as above. With this procedure we obtain an extended Kleinian group $K_{3}$ (a subgroup of $K$ ). So far, this extended Kleinian group $K_{3}$ is constructed as in Theorem 2.1, so it is an extended Schottky group.

Let us now assume the $K$-stabilizer of a boundary loop $C_{3}$ to be trivial. Then the projection loop $Q\left(P\left(C_{3}\right)\right)$ is a simple loop in the interior of $S$ so that if we cut $S$ along $Q\left(P\left(C_{3}\right)\right)$ then we obtain two extra boundaries. These two extra boundaries correspond to two structure loops in the boundary of $\mathcal{D}$; one of them being $C_{3}$. There is then an element $h \in K$ so that $h\left(C_{3}\right)$ is the other boundary loop which projects to $Q\left(P\left(C_{3}\right)\right)$. That boundary loop is different from $C_{3}$ as the $K$-stabilizer of $C_{3}$ was assumed to be trivial. In this way there is no option that $h$ is either loxodromic or a glide-reflection. We have performed part (2), that is, an HNN-extension, in Klein-Maskit's combination 
theorem 3.1 for $K_{3}$ using $h$. With this type of procedure, we obtain an extended Kleinian group $K_{4}$ (again a subgroup of $K$ ). Let us note that by the choice of $\mathcal{D}$ we necessarily have $\Omega\left(K_{4}\right) / K_{4}=S$. It follows that $K=K_{4}$.

At this point we are almost done. The only problem is that when performing the last HNN-extensions we may have situations as already described in Remark 2.2. But, as already noticed in both cases of Remark, we may proceed to modify some of the elements used in the HNN-extension to obtain the desired result.

5. Proof of Theorem 2.5. We make suitable modifications to the arguments given in [5] to obtain the desired result. Let us consider the canonical projection $\theta: \widehat{K} \rightarrow$ $G=\widehat{K} / \Gamma$ and let $\theta\left(\eta_{i}\right)=\sigma \in G$. If $\Omega$ is the region of discontinuity of $\left.\widehat{K}\right)$, then, as $\Gamma$ has finite index in $\widehat{K}, \Omega$ is also the region of discontinuity of $\Gamma$.

The group $K_{\sigma}=\theta^{-1}(\langle\sigma\rangle)$ turns out to be a an extended Schottky group. By the decomposition structure provided by Theorem $2.1, K_{\sigma}$ is constructed using $a$ cyclic groups generated by reflections, $b$ cyclic groups generated by imaginary reflections, $e$ real Schottky groups and some finite number of cyclic groups generated by either loxodromic transformations or glide-reflections.

Let us first consider the imaginary reflections of $K_{\sigma}$. The arguments for reflections are similar. Each imaginary reflection in $K_{\sigma}$ is conjugate in $\widehat{K}$ to some of the canonical imaginary reflection $\eta_{j}$. Two imaginary reflections in $K_{\sigma}$ provide different fixed points of $\sigma$ if they are non-conjugate in $K_{\sigma}$. It follows that we need to count the imaginary reflections of the form $w \eta_{j} w^{-1}$ that belong to $K_{\sigma}$ which are non-conjugate in $K_{\sigma}$. For, set first $C_{i}=\theta^{-1}(\mathrm{C}(\widehat{G}, \sigma))$ and note that $C_{i}$ normalizes $K_{\sigma}$. Indeed, if $w \in C_{i}$, then $\theta(w)$ commutes with $\sigma$. Now, let $\gamma \in K_{\sigma}$. As $\theta(\gamma) \in\langle\sigma\rangle, \theta(w)$ commutes with $\theta(\gamma)$. It follows that $\theta\left(w^{-1} \gamma w\right)=\theta(w)^{-1} \theta(\gamma) \theta(w)=\theta(\gamma) \in\langle\sigma\rangle$, that is, $w^{-1} \gamma w \in K_{\sigma}$.

Note that for $w \in \widehat{K}$, we have $w \eta_{i} w^{-1} \in K_{\sigma}$ if and only if $\theta(w) \sigma \theta(w)^{-1}=\sigma$ which is equivalent to have $w \in C_{i}$. Now for $w_{1}, w_{2} \in C_{i}, w_{1} \eta_{i} w_{1}^{-1}$ is conjugate in $K_{\sigma}$ to $w_{2} \eta_{i} w_{2}^{-1}$ if and only if there is some $\gamma \in K_{\sigma}$ so that $w_{1}^{-1} \gamma w_{2} \in \mathrm{C}\left(\widehat{K}, \eta_{i}\right)$. As $w_{1}^{-1} \gamma w_{2}=$ $\left(w_{1}^{-1} w_{2}\right)\left(w_{2}^{-1} \gamma w_{2}\right)$, the above is equivalent to have $w_{1} w_{2}^{-1} \in \mathrm{C}\left(\widehat{K}, \eta_{i}\right) K_{\sigma}$.

It follows from above that the conjugates of $\eta_{i}$ give rise to $n_{i}$ fixed points of $\sigma$, where

$$
\begin{aligned}
n_{i} & =\left[C_{i}: \mathrm{C}\left(\widehat{K}, \eta_{i}\right) K_{\sigma}\right] \\
& =\left[\theta\left(C_{i}\right): \theta\left(\mathrm{C}\left(\widehat{K}, \eta_{i}\right)\right) \theta\left(K_{\sigma}\right)\right] \\
& =\left[\mathrm{C}(\widehat{G}, \sigma): \theta\left(\mathrm{C}\left(\widehat{K}, \eta_{i}\right)\right)\langle\sigma\rangle\right] .
\end{aligned}
$$

As $\sigma \in \theta\left(\mathrm{C}\left(\widehat{K}, c_{i}\right)\right)$, we obtain that

$$
n_{i}=\left[\mathrm{C}(\widehat{G}, \sigma): \theta\left(\mathrm{C}\left(\widehat{K}, \eta_{i}\right)\right)\right]
$$

Now for $w \in \widehat{K}$, let $w \eta_{j} w^{-1} \in K_{\sigma}$, that is, $\theta(w) \theta\left(\eta_{j}\right) \theta(w)^{-1}=\sigma$. There is some $\gamma_{1} \in \Gamma$ for which $w \eta_{j} w^{-1}=\eta_{i} \gamma_{1}$. So there is some $\gamma_{2} \in \Gamma$ so that $\eta_{j}=w^{-1} \eta_{i} w \gamma_{2}$ (since $\Gamma$ is a normal subgroup in $\widehat{K})$. It follows that for each $v \in \widehat{K}$ we have $v \eta_{j} v^{-1}=$ $\left(v w^{-1} \eta_{i} w v^{-1}\right)\left(v \gamma_{2} v^{-1}\right)$. Therefore, $v \eta_{j} v^{-1} \in K_{\sigma}$ if and only if $v w^{-1} \eta_{i} w v^{-1} \in K_{\sigma}$ or equivalently $v w^{-1} \in C_{i}$.

Let $u_{1}, u_{2} \in C_{i}, \quad v_{1}=u_{1} w$ and $v_{2}=u_{2} w$. The imaginary reflections $v_{1} \eta_{j} v_{1}^{-1}, v_{2} \eta_{j} v_{2}^{-1} \in K_{\sigma}$ are conjugate in $K_{\sigma}$ if and only if $u_{1}^{-1} u_{2} \in w\left(\mathrm{C}\left(\widehat{K}, \eta_{j}\right) \Gamma\right) w^{-1}$. In fact, $v_{1} \eta_{j} v_{1}^{-1}$ and $v_{2} \eta_{j} v_{2}^{-1}$ are conjugate in $K_{\sigma}$ if and only if there is some 
$\gamma \in K_{\sigma} \quad$ so that $v_{1}^{-1} \gamma v_{2} \in \mathrm{C}\left(\widehat{K}, \eta_{j}\right)$. As $v_{2}^{-1} \gamma v_{2}=w^{-1}\left(u_{2}^{-1} \gamma u_{2}\right) w \in w^{-1} K_{\sigma} w=$ $K_{j}=\theta^{-1}\left(\left\langle\theta\left(\eta_{j}\right)\right\rangle\right)=\left\langle\Gamma, \eta_{j}\right\rangle$, we have that $v_{1} \eta_{j} v_{1}^{-1}$ and $v_{2} \eta_{j} v_{2}^{-1}$ are conjugates in $K_{\sigma}$ if and only if $v_{1}^{-1} v_{2} \in \mathrm{C}\left(\widehat{K}, \eta_{j}\right) K_{j}=\mathrm{C}\left(\widehat{K}, \eta_{j}\right) \Gamma$. This asserts that $u_{1}^{-1} u_{2}=w\left(v_{1}^{-1} v_{2}\right) w^{-1} \in w\left(\mathrm{C}\left(\widehat{K}, \eta_{j}\right) \Gamma\right) w^{-1}$ as desired.

If $C_{j}=\theta^{-1}\left(\mathbf{C}\left(\widehat{G}, \theta\left(\eta_{j}\right)\right)\right)$, then as $w C_{j} w^{-1}=C_{i}$, the above asserts that given $j \in I(i)$ conjugations of $\eta_{j}$ in $\widehat{K}$ produce in $K_{\sigma}, n_{j}=\left[C_{j}: \mathrm{C}\left(\widehat{K}, \eta_{j}\right) \Gamma\right]$ isolated fixed points of $\sigma$. Note that

$$
\begin{aligned}
n_{j} & =\left[C_{j}: \mathrm{C}\left(\widehat{K}, \eta_{j}\right) \Gamma\right] \\
& =\left[C_{j} / \Gamma: \mathrm{C}\left(\widehat{K}, \eta_{j}\right) \Gamma / \Gamma\right] \\
& =\left[\mathrm{C}\left(\widehat{G}, \theta\left(\eta_{j}\right)\right): \theta\left(\mathrm{C}\left(\widehat{K}, \eta_{j}\right)\right)\right] .
\end{aligned}
$$

Finally, with the observation that for $k, l \in J(i)$, the imaginary reflections $\eta_{k}$ and $\eta_{l}$ are non-conjugate in $K_{\sigma}$, all the above provides the formula that permits to count the number $b$ of non-conjugated imaginary reflections in $K_{\sigma}$.

The arguments for the case of reflections and real Schottky groups follow the same lines as the above case and so we omit it. This finishes the proof of Theorem 2.5.

6. Algebraic types of extended Schottky groups. As a consequence of Theorem 2.1 and its proof, one may easily describe the algebraic structures of extended Schottky groups and their uniformized orbifolds. In this section we provide some observations as a consequence of that description.

6.1. Algebraic types. Let us consider an extended Schottky group $K$ of type

$$
\left(\xi ; b, c, d,\left\{p_{1}, \ldots, p_{r}\right\},\left\{q_{1}, \ldots, q_{s}\right\} ; a,\left\{k_{1}, \ldots, k_{r}\right\},\left\{l_{1}, \ldots, l_{s}\right\}\right) .
$$

The type asserts that $K$ is constructed as in Theorem 2.1 by using $a$ groups of type (i), $b$ groups of type (ii), $c$ groups of type (iii), $d$ groups of type (iv) and $e=r+s$ real Schottky groups of type $\left(+; p_{j} ; k_{j}\right)$ for $j=1, \ldots, r$, and of type $\left(-; q_{j} ; l_{l}\right)$ for $j=1, \ldots, s$. It now follows, from Klein-Maskit's combination theorem, that $K$ is isomorphic as an abstract group to

$$
\left\{\begin{array}{c}
\underbrace{\mathrm{Z}_{2} * \cdots * \mathrm{Z}_{2}}_{a+b} * \underbrace{\mathrm{Z} * \cdots * \mathrm{Z}}_{c+d} * \\
*\left(\mathrm{Z}_{2} \oplus(\underbrace{\mathrm{Z} * \cdots * \mathrm{Z}}_{2 p_{1}+k_{1}-1})\right) * \cdots *\left(\mathrm{Z}_{2} \oplus(\underbrace{\mathrm{Z} * \cdots * \mathrm{Z}}_{2 p_{r}+k_{r}-1})\right) * . \\
*\left(\mathrm{Z}_{2} \oplus(\underbrace{\mathrm{Z} * \cdots * \mathrm{Z}}_{q_{1}+l_{1}-1})\right) * \cdots *\left(\mathrm{Z}_{2} \oplus(\underbrace{\mathrm{Z} * \cdots * \mathrm{Z}}_{q_{s}+l_{s}-1})\right)
\end{array}\right.
$$

Let $S=\Omega / K$ be the compact surface uniformized by $K$. The orientability type of $S$ is determined by $\xi \in\{ \pm\}$; the surface being orientable if and only if $\xi=+$. The first $(a+b)$ factors provide a compact surface $S_{1}$ homeomorphic to the connected sum of $b$ projective planes with $a$ boundary components. The second $(c+d)$ factors correspond to make a connected sum of $S_{1}$ with a closed orientable surface of genus $d$ and with 
the connected sum of $2 c$ real projective planes. Let us denote the resulting compact surface by the symbol $S_{2}$. The other factors provide the type of real Schottky groups to use in the construction. Each factor $\mathrm{Z}_{2} \oplus(\underbrace{\mathrm{Z} * \cdots * \mathrm{Z}}_{2 p_{j}+k_{j}-1})$ provides a Riemann surface of genus $p_{j}$ and also $k_{j}$ boundary components with which to make a connected sum with $S_{2}$. Each factor $\mathrm{Z}_{2} \oplus(\underbrace{\mathrm{Z} * \cdots * \mathrm{Z}}_{q_{j}+l_{j}-1})$ provides a connected sum of $q_{j}$ real projective planes and $l_{j}$ boundary components to make a connected sum with the previous compact (bordered) surface.

Summarizing the above we have the following.

Proposition 6.1. If $K$ is an extended Schottky group, with region of discontinuity $\Omega$, of type

$$
\left(+; 0,0, d,\left\{p_{1}, \ldots, p_{r}\right\},\{-\} ; a,\left\{k_{1}, \ldots, k_{r}\right\},\{-\}\right),
$$

then $\Omega / K$ is a compact Riemann surface of genus $d+p_{1}+\cdots+p_{r}$ with exactly $a+$ $k_{1}+\cdots+k_{r}$ boundary components. Similarly, if $K$ is an extended Schottky group, with region of discontinuity $\Omega$, of type

$$
\left(-; b, c, d,\left\{p_{1}, \ldots, p_{r}\right\},\left\{q_{1}, \ldots, q_{s}\right\} ; a,\left\{k_{1}, \ldots, k_{r}\right\},\left\{l_{1}, \ldots, l_{s}\right\}\right),
$$

then $\Omega / K$ is a compact non-orientable Klein surface of topological genus $b+2 c+2 d+$ $2 p_{1}+\cdots+2 p_{r}+q_{1}+\cdots+q_{s}$ with exactly $a+k_{1}+\cdots+k_{r}+l_{1}+\cdots+l_{s}$ boundary components.

Proof. This follows from the structural picture in Theorem 2.1 together with previous observations.

6.2. Isomorphisms classes of extended Schottky groups. Clearly, two extended Schotky groups of different orientability type cannot be conjugated topologically, but they may be isomorphic as abstract groups; for instance, geometrically extended Schottky groups of types

$$
(+; 0,0,0,\{-\},\{-\} ; 2,\{-\},\{-\}), \text { and }(-; 2,0,0,\{-\},\{-\} ; 0,\{-\},\{-\}),
$$

are isomorphic to $\mathrm{Z}_{2} * \mathrm{Z}_{2}$. The first type gives uniformizations of a closed annulus and the second one gives uniformizations of the Klein bottle.

Proposition 6.1 provides the topological structure for the (bordered) compact surface uniformized by an extended Schottky group of a given type. In particular, we may decide, in terms of the types, when two extended Schottky groups uniformize topologically equivalent compact surfaces. For it, their types should provide the same orientability type, the same topological genus of the surface and the same number of boundary components. We proceed to list all of the possibilities.

Proposition 6.2 (Orientable type). Let $K_{j}$ be an orientable extended Schottky group of type

$$
\left(+; 0,0, d_{j},\left\{p_{1}^{j}, \ldots, p_{r_{j}}^{j}\right\},\{-\} ; a_{j},\left\{k_{1}^{j}, \ldots, k_{r_{j}}^{j}\right\},\{-\}\right), \quad j=1,2 .
$$


(a) $K_{1}$ and $K_{2}$ uniformize topologically equivalent compact Riemann surfaces if and only if

$$
\left(*_{1}\right)\left\{\begin{array}{l}
d_{1}+p_{1}^{1}+\cdots+p_{r_{1}}^{1}=d_{2}+p_{1}^{2}+\cdots+p_{r_{2}}^{2} \\
a_{1}+k_{1}^{1}+\cdots+k_{r_{1}}^{1}=a_{2}+k_{1}^{2}+\cdots+k_{r_{2}}^{2}
\end{array} .\right.
$$

(b) Under the equality $\left(*_{1}\right)$ we have that $K_{1}$ and $K_{2}$ are isomorphic as abstract groups if and only if

$$
\left(*_{2}\right)\left\{\begin{array}{l}
a_{1}=a_{2}=a \\
d_{1}=d_{2}=d \\
r_{1}=r_{2}=r
\end{array}\right.
$$

and there exists a permutation $\sigma \in \Sigma_{r}$ so that

$$
\left(*_{3}\right)\left\{\begin{array}{l}
k_{j}^{1}=k_{\sigma(j)}^{2} \\
p_{j}^{1}=p_{\sigma(j)}^{2}
\end{array}\right.
$$

(c) The extended Schottky groups $K_{1}$ and $K_{2}$ are topologically equivalent if and only if the three equalities $\left(*_{1}\right),\left(*_{2}\right)$ and $\left(*_{3}\right)$ hold simultaneously.

Proof. By Proposition 6.1 the surface uniformized by $K_{j}$ is a compact Riemann surface of genus $g_{j}=d_{j}+p_{j}^{j}+\cdots+p_{r_{j}}^{j}$ bordered by $a_{j}+k_{1}^{j}+\cdots+k_{r_{j}}^{j}$ boundary components. Then $\left(*_{1}\right)$ follows from equality of genus and number of boundary components. Equality $\left(*_{2}\right)$ follows directly from the algebraic structure given in (1). Part (c) follows from the fact that the basic groups to use in the construction of both extended Schottky groups (in Theorem 2.1) should be in the same number for each possible type.

Proposition 6.3 (Non-orientable type). Let $K_{j}$ be a non-orientable extended Schottky group of type

$$
\left(-; b_{j}, c_{j}, d_{j},\left\{p_{1}^{j}, \ldots, p_{r_{j}}^{j}\right\},\left\{q_{1}^{j}, \ldots, q_{s_{j}}^{j}\right\} ; a_{j},\left\{k_{1}^{j}, \ldots, k_{r_{j}}^{j}\right\},\left\{l_{1}^{j}, \ldots, l_{s_{j}}^{j}\right\}\right), \quad j=1,2 .
$$

(a) $K_{1}$ and $K_{2}$ uniformize topologically equivalent compact non-orientable Klein surfaces if and only if

$$
\left(* *_{1}\right)\left\{\begin{array}{c}
b_{1}+2 c_{1}+2 d_{1}+2 p_{1}^{1}+\cdots+2 p_{r_{1}}^{1}+q_{1}^{1}+\cdots+q_{s_{1}}^{1}= \\
b_{2}+2 c_{2}+2 d_{2}+2 p_{1}^{2}+\cdots+2 p_{r_{2}}^{2}+q_{1}^{2}+\cdots+q_{s_{2}}^{2} . \\
a_{1}+k_{1}^{1}+\cdots+k_{r_{1}}^{1}+l_{1}^{1}+\cdots+l_{s_{1}}^{1}=a_{2}+k_{1}^{2}+\cdots+k_{r_{2}}^{2}+l_{1}^{2}+\cdots+l_{s_{2}}^{2}
\end{array}\right.
$$

(b) Under the equality $\left(* *_{1}\right)$ we have that $K_{1}$ and $K_{2}$ are isomorphic as abstract groups if and only if

$$
\left(* *_{2}\right)\left\{\begin{array}{l}
a_{1}+b_{1}=a_{2}+b_{2} \\
c_{1}+d_{1}=c_{2}+d_{2} \\
r_{1}+s_{1}=r_{2}+s_{2}
\end{array}\right.
$$


and there is a permutation $\sigma \in \Sigma_{u}$ so that

$$
\left(* *_{3}\right)\left\{\begin{array}{l}
\text { (1) if } j \in\left\{1, \ldots, r_{1}\right\} \text { and } \sigma(j) \in\left\{1, \ldots, r_{2}\right\}, \text { then } \\
\quad 2 p_{j}^{1}+k_{j}^{1}=2 p_{\sigma(j)}^{2}+k_{\sigma(j)}^{2} ; \\
\text { (2) if } j \in\left\{1, \ldots, r_{1}\right\} \text { and } \sigma(j) \in\left\{r_{2}+1, \ldots, r_{2}+s_{2}\right\}, \text { then } \\
\quad 2 p_{j}^{1}+k_{j}^{1}=q_{\sigma(j)-r_{2}}^{2}+l_{\sigma(j)-r_{2}}^{2} ; \\
\text { (3) if } j \in\left\{r_{1}+1, \ldots, r_{1}+s_{1}\right\} \text { and } \sigma(j) \in\left\{1, \ldots, r_{2}\right\}, \text { then } \\
\quad q_{j-r_{1}}^{1}+l_{j-r_{1}}^{1}=2 p_{\sigma(j)}^{2}+k_{\sigma(j)}^{2} ; \\
\text { (4) if } j \in\left\{r_{1}+1, \ldots, r_{1}+s_{1}\right\} \text { and } \sigma(j) \in\left\{r_{2}+1, \ldots, r_{2}+s_{2}\right\}, \text { then } \\
\quad q_{j-r_{1}}^{1}+l_{j-r_{1}}^{1}=q_{\sigma(j)-r_{2}}^{2}+l_{\sigma(j)-r_{2}}^{2}
\end{array}\right.
$$

(c) The extended Schottky groups $K_{1}$ and $K_{2}$ are topologically equivalent if and only if the three equalities $\left(* *_{1}\right),\left(* *_{2}\right)$ and $\left(* *_{3}\right)$ hold simultaneously.

Proof. The idea is similar as to the orientable case.

7. Connection to symmetries of handlebodies. As already mentioned in the Abstract and the Introduction, the structural description of extended Schottky groups provides alternative arguments to obtain the results in Kalliogis-McCullough [13]. In this last section, as a matter of completeness, we provide the following relations. A compact Kleinian three-manifold $M$ is a compact three-manifold whose interior $M^{0}$ carries a complete hyperbolic structure and its boundary $S$ carries a natural structure of a closed Riemann surface (maybe not connected), which is the conformal boundary of the hyperbolic structure. In other words, there is a torsion-free Kleinian group $\Gamma$ with the region of discontinuity $\Omega \subset \widehat{\mathbb{C}}$ (maybe empty) so that $M=\left(\mathbb{H}^{3} \cup \Omega\right) / \Gamma$. In this case, $M^{0}=\mathbb{M}^{3} / \Gamma$ and $S=\Omega / \Gamma$. A symmetry of $M$ is an orientation-reversing selfhomeomorphism of order two whose restriction to the interior of $M$ is a hyperbolic isometry. The restriction of a symmetry of $M$ to its conformal boundary $S$ is a symmetry of $S$ but not every symmetry of $S$ may necessarily be obtained by the restriction of a symmetry of $M$.

Let $M=\left(\mathbb{H}^{3} \cup \Omega\right) / \Gamma$ be a compact Kleinian three-manifold homeomorphic to a handlebody, say of genus $g$, and let $S=\Omega / \Gamma$ be its conformal boundary (a closed Riemann surface of genus $g$ ). In this case $\Gamma$ is a Schottky group of rank $g$. If $\tau$ is a symmetry of $M$, then by lifting it to the universal cover $\mathbb{H}^{3} \cup \Omega$, we obtain an extended Schottky group $K$ whose orientation-preserving half is $\Gamma$. It follows from Theorem 2.1 that each connected component of the set of fixed points of $\tau$ is either a point (the projection of the fixed point of imaginary reflections) or a two-dimensional compact surface (coming from the reflections or the real Schottky factors) which may be orientable or not and that $\tau$ has at most $g+1$ connected components of the set of fixed points (this was already pointed in [13] using three-dimensional arguments together with combinatorial techniques). The quotient $M /\langle\tau\rangle$ fails to be a manifold exactly at the projection of the isolated fixed points of $\tau$ (locally looks like a cone over the projective plane) and its boundary carries a natural compact Klein surface structure. At this point it is important to note that the presence of isolated fixed points 
is not detected by the restriction of $\tau$ to the conformal boundary $S$ and so the above bound is not a direct consequence of Harnack's result. Next, we summarize the above.

COROLlaRY 7.1. Let $K$ be an extended Schottky group constructed as in Theorem 2.1 using $\alpha$ groups of type (i), $\beta$ groups of type (ii), $\gamma$ groups of type (iii), $\delta$ groups of type (iv) and $\varepsilon$ groups of type (v). If $\Gamma$ is the canonical orientation-preserving subgroup of $K$ and $M=\left(\mathbb{M}^{3} \cup \Omega\right) / \Gamma$, then $K / \Gamma$ represents a symmetry of $M$ whose connected components of fixed points consist of $\alpha$ two-dimensional closed discs, $\beta$ isolated points, and $\varepsilon$ twodimensional non-simply connected compact surfaces. In particular, if $M$ is a handlebody of genus $g$ with a Schottky structure and $\tau: M \rightarrow M$ is a symmetry with $n_{0}$ isolated fixed points, $n_{1}$ total ovals in the conformal boundary and $n_{2}$ two-dimensional connected components of the set of fixed points, then

$$
\begin{aligned}
& 0 \leq n_{0}+n_{1} \leq g+1, \\
& 0 \leq n_{0}+n_{2} \leq g+1 .
\end{aligned}
$$

As handlebodies are compression bodies, given a tame orientation-reversing involution $\tau$ on a handlebody $M$, there is an extended Schottky group $K$ so that there is an orientation-preserving homeomorphism $h: M t o M_{\Gamma}$, where $\Gamma=K^{+}$, so that $h \tau h^{-1}$ is the anti-conformal involution induced by $K$ (see also [32]). As the orientation-preserving half is unique, this asserts that the number of topologically different extended Schottky groups is equal to the number of topological classes of tame orientation-reversing involutions on a handlebody. In particular, Corollary 2.6 provides an upper bound. By group-theoretic methods such an enumeration has been obtained by Stephanus [31].

ACKNOWLEDGEMENTS. The authors would like to thank the referee for his/her careful reading and the suggested corrections to the first version. The first author was partially supported by the Research Grant N201 366436 of the Polish Ministry of Science and Higher Education and the second one by Projects Fondecyt 1110001 and UTFSM 12.11.01.

\section{REFERENCES}

1. L. Ahlfors and L. Sario, Riemann surfaces (Princeton University Press, Princeton NJ, 1960).

2. N. Alling and N. Greenleaf, Foundations of the theory of Klein surfaces, Lecture Notes in Mathematics, vol. 219 (Springer, Berlin, Germany, 1971).

3. E. Bujalance, J. F. Cirre, J. M. Gamboa and G. Gromadzki, Symmetries of compact Riemann surfaces, Lecture Notes in Mathematics vol. 2007 (Springer-Verlag, Berlin, Germany, 2010).

4. L. Greenberg, Maximal Fuchsian Groups. Bull. AMS 69 (1963), 569-573.

5. G. Gromadzki, On a Harnack-Natanzon theorem for the family of real forms of Riemann surfaces, J. Pure Appl. Algebra 121 (1997), 253-269.

6. A. Harnack, Ueber die Vieltheiligkeit der ebenen algebraischen Curven, Math. Ann. 10(2) (1876), 1189-198.

7. B. Heltai, Symmetric Riemann surfaces, torsion subgroups and Schottky coverings, Proc. Am. Math. Soc. 100 (1987), 675-682. 
8. R. A. Hidalgo, Automorphisms groups of Schottky type, Ann. Acad. Sci. Fenn. 30 (2005), 183-204. $141-158$.

9. R. A. Hidalgo, Maximal Schottky extension groups, Geometriae Dedicata 146(1) (2010),

10. R. A. Hidalgo, An equivariant loop theorem for Kleinian groups: A decomposition structure of extended function groups (Preprint).

11. R. A. Hidalgo and B. Maskit, On Klein-Schottky groups, Pacific J. Math. 220(2) (2005), 313-328.

12. R. A. Hidalgo and B. Maskit, A note on the lifting of automorphisms. in Geometry of Riemann surfaces. Lecture Notes of the London Mathematics Society, vol. 368 (Fred G., Gabino G. and Christos K., Editors) (Cambridge University Press, Cambridge, UK, 2010), 260-267. ISBN-13-9780521733076.

13. J. Kalliongis and D. McCullough, Orientation-reversing involutions on handlebodies, Trans. Math. Soc. 348(5) (1996), 1739-1755.

14. F. Klein, Über Realitätsverhältnisse bei der einem beliebigen Geschlechte zugehörigen Normalkurve der $\phi$. Math. Annalen 42(1) (1893), 1-29.

15. A. M. Macbeath, The classification of non-Euclidean crystallographic groups, Canad. J. Math. 19 (1967), 1192-1205.

16. A. M. Macbeath and D. Singerman, Spaces of subgroups and Teichmüller space, Proc. Lond. Math. Soc. 31(3) (1975), 211-256.

17. B. Maskit, Construction of Kleinian groups, in Proc. of the conf. on complex anal., Minneapolis, 1964 (Aeppli A., Calabi E. and Röhrl H., Ediors) (Springer-Verlag, Berlin, Germany, 1965), 281-296.

18. B. Maskit, A characterization of Schottky groups, J. d'Analyse Math. 19 (1967), 227 230.

19. B. Maskit, The conformal group of a plane domain, Amer. J. Math. 90(3) (1968), $718-722$.

20. B. Maskit, On Klein's combination theorem III. Advances in the theory of Riemann surfaces (Proc. of Conf., Stony Brook, NY, 1969), Ann. Math. Stud. 66(1971), 297-316(Princeton University Press).

21. B. Maskit, Decomposition of certain Kleinian groups, Acta Math. 130 (1973), 243-263.

22. B. Maskit, On the classification of Kleinian groups. I. Koebe groups, Acta Math. 135 (1975), 249-270.

23. B. Maskit, On the classification of Kleinian Groups. II. Signatures, Acta Math. 138 (1976), 17-42.

24. B. Maskit, Kleinian groups GMW (Springer-Verlag, Berlin, Germany, 1987).

25. B. Maskit, On Klein's combination theorem. IV. Trans. Am. Math. Soc. 336 (1993), $265-294$.

26. K. Matsuzaki and M. Taniguchi, Hyperbolic manifolds and Kleinina groups, Oxford Mathematical Monographs, Oxford Science Publications (Clarendon Press, Oxford University Press, New York, 1998).

27. W. H. Meeks III and S.-T. Yau, Topology of three-dimensional manifolds and the embedding problem in minimal surface theory. Ann. Math. 112(2) (1980), 441-484.

28. S. M. Natanzon, Differential equations for Prym theta-functions. A criterion for twodimensional finite zone potential Schrödinger operators to be real, Funktsional Anal. i Prilozhen 26 (1992), 17-26. (English translation in Funct. Anal. Appl. 26 (1992), 13-20.)

29. R. J. Sibner, Symmetric Fuchsian groups, Am. J. Math. 90 (1968), 1237-1259. $29-38$.

30. D. Singerman, Finitely maximal Fuchsian groups, J. Lond. Math. Soc. 6(2) (1972),

31. M. Stephanus, Orientation reversing involutions of a handlebody Dissertation (St. Louis University, St. Louis, MO, 1995).

32. B. Zimmermann, Über Homöomorphismen n-dimensionaler Henkelkörper und endliche Erweiterungen von Schottky-Gruppen (German), [On homeomorphisms of $n$ dimensional handlebodies and on finite extensions of Schottky groups] Comment. Math. Helv. 56 (1981), no. 3, 474-486.

33. B. Zimmermann, Finite maximal orientation reversing group actions on handlebodies and 3-manifolds, Rend. Circ. Mat. Palermo (2) 48(3) (1999), 549-562. 